\title{
SNMMI Procedure Standard/EANM Guideline for Gated Equilibrium Radionuclide Angiography*
}

\author{
Mary Beth Farrell, CNMT, NCT FASNC FSNMMI-TS ${ }^{1}$, James R. Galt, PhD ${ }^{2}$, Panagiotis Georgoulias, MD, PhD (Co-chair, \\ EANM) ${ }^{3}$, Saurabh Malhotra, FACC FASNC (Chair, SNMMI) ${ }^{4}$, Robert Pagnanelli, BSRT(R)(N), CNMT, NCT FASNC \\ FSNMMI-TS ${ }^{5}$, Christoph Rischpler, $\mathrm{MD}^{6}$, and Bital Savir-Baruch, $\mathrm{MD}^{7}$ \\ ${ }^{1}$ Intersocietal Accreditation Commission, Ellicott City, Maryland; ${ }^{2}$ Department of Radiology and Imaging Sciences, Emory University \\ School of Medicine, Atlanta, Georgia; ${ }^{3}$ Department of Nuclear Medicine, University of Thessaly, Larissa, Greece; ${ }^{4}$ Division of \\ Cardiology, Cook County Health and Rush Medical College, Chicago, Illinois; ${ }^{5}$ Department of Radiology, Duke Regional Hospital, \\ Durham, North Carolina; ${ }^{6}$ Department of Nuclear Medicine, University Hospital Essen, University of Duisburg-Essen, Essen, \\ Germany; and ${ }^{7}$ Department of Radiology, Loyola University Medical Center, Maywood, Illinois
}

The purpose of this document is to assist nuclear medicine practitioners in recommending, performing, interpreting, and reporting the results of gated equilibrium radionuclide angiocardiography (ERNA).

J Nucl Med Technol 2020; 48:126-135

DOI: 10.2967/jnmt.120.246405

\section{PREAMBLE}

The Society of Nuclear Medicine and Molecular Imaging (SNMMI) is an international scientific and professional organization founded in 1954 to promote the science, technology, and practical application of nuclear medicine.

The European Association of Nuclear Medicine (EANM) is a professional nonprofit medical association that facilitates communication worldwide between individuals pursuing clinical and research excellence in nuclear medicine. The EANM was founded in 1985. SNMMI and EANM members are physicians, technologists, and scientists specializing in the research and practice of nuclear medicine.

The SNMMI and EANM will periodically define new guidelines for nuclear medicine practice to help advance the science of nuclear medicine and to improve the quality of service to patients throughout the world. Existing practice guidelines will be reviewed for revision or renewal, as appropriate, on their fifth anniversary or sooner, if indicated.

\footnotetext{
Received Apr. 1, 2020; accepted Apr. 3, 2020.

For correspondence or reprints contact: Saurabh Malhotra, Director of Advanced Cardiac Imaging, Cook County Health and Hospitals System, Assistant Professor of Medicine (Cardiology), Rush Medical College, John H. Stroger Hospital of Cook County, 1901 W. Harrison Street, Suite 3620, Chicago, IL 60612.

E-mail: saurabh.malhotra@cookcountyhhs.org

"NOTE: FOR CE CREDIT, YOU CAN ACCESS THIS ACTIVITY THROUGH THE

SNMMI WEB SITE (http://www.snmmi.org/ce_online) THROUGH JUNE 2023.

COPYRIGHT (c) 2020 by the Society of Nuclear Medicine and Molecular Imaging.
}

Each practice guideline, representing a policy statement by the SNMMI/EANM, has undergone a thorough consensus process in which it has been subjected to extensive review. The SNMMI and EANM recognize that the safe and effective use of diagnostic nuclear medicine imaging requires specific training, skills, and techniques, as described in each document. Reproduction or modification of the published practice guideline by those entities not providing these services is not authorized.

These guidelines are an educational tool designed to assist practitioners in providing appropriate care for patients. They are not inflexible rules or requirements of practice and are not intended, nor should they be used, to establish a legal standard of care. For these reasons and those set forth below, both the SNMMI and the EANM caution against the use of these guidelines in litigation in which the clinical decisions of a practitioner are called into question.

The ultimate judgment regarding the propriety of any specific procedure or course of action must be made by the physician or medical physicist in light of all the circumstances presented. Thus, there is no implication that an approach differing from the guidelines, standing alone, is below the standard of care.

To the contrary, a conscientious practitioner may responsibly adopt a course of action different from that set forth in the guidelines when, in the reasonable judgment of the practitioner, such course of action is indicated by the condition of the patient, limitations of available resources, or advances in knowledge or technology subsequent to publication of the guidelines.

The practice of medicine includes both the art and the science of the prevention, diagnosis, alleviation, and treatment of disease. The variety and complexity of human conditions make it impossible to always reach the most appropriate diagnosis or to predict with certainty a particular response to treatment. Therefore, it should be recognized that adherence to these guidelines will not ensure an accurate diagnosis or a successful outcome.

All that should be expected is that the practitioner will follow a reasonable course of action based on current 
knowledge, available resources, and the needs of the patient to deliver effective and safe medical care. The sole purpose of these guidelines is to assist practitioners in achieving this objective.

\section{PURPOSE}

The purpose of this document is to assist nuclear medicine practitioners in recommending, performing, interpreting, and reporting the results of gated ERNA.

\section{BACKGROUND}

Gated ERNA is an imaging technique used to evaluate global and regional measures of ventricular function at rest or during stress, and to assess cardiac chamber morphology. Evaluation of ventricular function includes measurement of ventricular volumes and ejection fraction (EF) and assessment of ventricular wall motion and diastolic function. The procedure involves radiolabeling of patient's red blood cells (RBCs), followed by electrocardiogram (ECG)-gated bloodpool scintigraphy. Though mainly performed for the evaluation of left ventricular (LV) function, right ventricular (RV) function can also be evaluated. In routine clinical practice, ERNA is performed by a gated planar acquisition of the ventricles, though SPECT ERNA can also be performed. SPECT ERNA has the benefit of isolating the left and right ventricles, improving the evaluation of individual ventricular function (1). Particularly, after the introduction of cadmiumzinc-telluride (CZT) cameras, the imaging time for SPECT ERNA can be significantly reduced (2). Alternative terminologies for this technique include gated cardiac blood-pool imaging, multigated acquisition, radionuclide ventriculography, and gated radionuclide angiography (RNA).

\section{INDICATIONS}

The majority of ERNA studies are performed for assessment of LV volume and function at rest, with serial evaluation for monitoring of medical or surgical therapy, or to diagnose chemotherapy-associated cardiotoxicity (Table 1). In general, main indications for ERNA include applications in cardiooncology, valvular heart disease, and cardiomyopathy. The assessment of RV function may be performed before LV assist device implantation as RV heart failure is a common and major complication. Stress ERNA can also be performed for diagnosis of CAD (with exercise or dobutamine), and submaximal exercise stress ERNA has demonstrated prognostic value within 2 weeks after myocardial infarction. However, stress ERNA is often fraught with errors resulting from patient motion and change in positioning between rest and stress acquisitions. Additionally, when posttreadmill images are acquired, true peak stress left ventricular ejection fraction (LVEF) cannot be recorded. Due to limitations in diagnostic performance, stress ERNA is limited to research settings and rare clinical circumstances, and has largely been replaced by stress echocardiography and stress myocardial perfusion imaging in contemporary
TABLE 1

Indications for ERNA

Valvular heart disease

Timing of surgery

Assessment of treatment effect

Cardiomyopathy

Evaluation of biventricular function

Determination of type (systolic vs. diastolic) and severity

Assessment of diastolic dysfunction

Identification of candidates for defibrillator implantation and resynchronization therapy

Evaluation of ventricular function before and after transplantation

Cardio-oncology

Monitoring LV function during chemotherapy

Diagnosis of cardiotoxicity from chemotherapy

Guiding chemotherapy

Uncommon indications

Stable coronary artery disease*

Diagnosis (rest/stress ERNA)

Prognosis

Assessment of treatment efficacy

Adjunctive evaluations

Evaluation of RV function

Determination of ventricular dyssynchrony

*Performance of stress ERNA (exercise or dobutamine) in the setting of acute coronary syndromes is not recommended.

clinical practice. Detailed information on the strength of the recommendations for various indications of ERNA can be obtained from the American College of Cardiology (ACC)/ American Heart Association (AHA)/American Society for Nuclear Cardiology (ASNC) $(3,4)$ or EANM/European Society of Cardiology (ESC) (5) guidelines on Clinical Use of Cardiac Radionuclide Imaging.

LVEF quantification by ERNA has been shown to have excellent reproducibility $(6,7)$, with inter- and intraobserver variability $<5 \%(8)$. As such, it is often used in serial assessment of LVEF to assess for cardiotoxicity among patients undergoing chemotherapy (primarily with anthracyclines). Guidelines for the use of anthracycline chemotherapy based on serial ERNA assessment of LVEF is provided in Table 2.

\section{PROCEDURE}

\section{A. Patient Evaluation and Test Preparation}

A review of medical history should be performed with careful attention to cardiac history such as congenital heart disease, old or recent myocardial infarction, cardiac arrhythmias, prior cardiac injuries, medications, and cardiac risk factors. Women undergoing testing should be asked questions pertaining to pregnancy and lactation. In pregnant women, other modalities such as echocardiography or cardiac MR should be preferentially used for assessment of cardiac function, and among lactating women, breast feeding will need to be interrupted for 12-24 hours after the study $(11,12)$. 
TABLE 2

Guideline for Serial Monitoring of LVEF by ERNA for Patients Undergoing Chemotherapy with Anthracyclines $(9,10)$

Normal LVEF at baseline ( $\geq 50 \%)$

Baseline ERNA before initiating chemotherapy

Next ERNA at $250-300 \mathrm{mg} / \mathrm{m}^{2}$ dose

Next ERNA at $450 \mathrm{mg} / \mathrm{m}^{2}\left(400 \mathrm{mg} / \mathrm{m}^{2}\right.$ if high risk $)$

Next ERNA before each dose $>450 \mathrm{mg} / \mathrm{m}^{2}$

Discontinue therapy if LVEF decreases $\geq 10 \%$ (EF units)

from baseline and reaches $<50 \%$

Abnormal LVEF at baseline ( $>30 \%$ to $<50 \%)$

Baseline ERNA before initiating chemotherapy

Serial ERNA before each subsequent dose

Discontinue therapy if LVEF decreases $\geq 10 \%$ (EF units)

from baseline or reaches $\leq 30 \%$

LVEF $\leq 30 \%$ at baseline

Do not initiate therapy

*High-risk features include use of cyclophosphamide, heart disease, mediastinal radiation, and abnormal ECG.

\section{Rest Images}

i. Electrode placement: Three limb lead electrodes are required, as follows: left arm-usually placed at the left upper chest; right arm-usually placed at the right upper chest; and left leg-usually at the left lower chest. As required for reliable gating with any form of gated imaging, lead placement should be optimized to emulate the lead from a recent standard 12-lead ECG that provides a high voltage monophasic $\mathrm{R}$ wave or reversed $\mathrm{S}$ wave QRS with relatively low amplitude $\mathrm{P}$ waves and $\mathrm{T}$ waves. Areas with significant hair may need to be shaved to reduce signal interruptions. It is important to confirm the synchronization of the ECG gating to the peak of an $\mathrm{R}$ wave. Poor quality or abnormal triggering can significantly alter the ventricular volume curve.

ii. Diet: No specific preparations are required. Fasting state is not required, though would be necessary if stress imaging is also being performed.

2. Stress Images

i. Informed consent should be signed after reviewing the risks of exercise or dobutamine stress test. In the case of dobutamine stress test, the patient should be asked about history of adverse reactions related to dobutamine.

ii. Electrode placement: 12-lead ECG monitoring must be performed using 3 limbs (as above) and 6 precordial leads (V1-V6).

iii. Diet: Patients should fast for 3-4 hours.

iv. Medications: It is recommended that cardiac medications (such as $\beta$-blockers, nitrates etc.) that may interfere with stress testing be withheld, in consultation with the referring physician. v. Safety: 12-lead ECG monitoring, life support and cardiac resuscitation instruments, medications for resuscitation, and trained medical personnel should be available.

\section{B. Precautions}

1. Stress Test Contraindications (bicycle and dobutamine) $(13,14)$ : Unstable angina, decompensated heart failure, systolic blood pressure above 200 and diastolic pressure above $110 \mathrm{~mm} \mathrm{Hg}$, uncontrolled arrhythmias, symptomatic aortic stenosis/dissection, acute pulmonary embolism, acute myocarditis, pericarditis or endocarditis, myocardial infarction in the past 4 days, known hypertrophic obstructive cardiomyopathy (for dobutamine stress), and severe pulmonary hypertension.

2. Tagging of Autologous RBC In Vitro: Per the Occupational Safety and Health Administration (OSHA) guidelines for safe handling of human blood products (www.osha.gov) to prevent potential transmission of blood-borne pathogens and USP 797, care must be taken to ensure that after labeling, the tagged RBCs are reinjected into the same patient from whom the blood was drawn (14). It is recommended that the laboratory develop a standardized procedure for identifying and documenting the correct patient, in a fashion similar to that used during blood transfusion. Although in vivo labeling can be performed, in vitro labeling is strongly recommended on account of its superior labeling efficiency.

\section{Stress Protocol}

1. Bicycle Ergometer: Examination is usually performed with a supine (most commonly) or upright bicycle ergometer. However, in patients who cannot perform adequate exercise, a positive inotropic agent such as dobutamine can be used.

2. Pharmaceutical: Dobutamine is the agent of choice. Vasodilator stress, which is typically used for myocardial perfusion imaging, has low accuracy for stress ERNA, and is rarely used (15).

3. Monitoring: Vitals should be taken at rest, at consistent intervals during stress, and during recovery, until they return to baseline or until any ECG changes have normalized.

\section{Radiopharmaceuticals}

1. Introduction: The most commonly used radiotracer for autologous RBC labeling is ${ }^{99 \mathrm{~m}} \mathrm{Tc}$-labeled RBC. ${ }^{99 \mathrm{~m} T c-l a b e l e d ~ h u m a n ~ s e r u m ~ a l b u m i n ~ r e s u l t s ~}$ in suboptimal image quality, when compared with labeled RBCs, due to faster breakdown of the albumin, and as such its use is not recommended.

i. Dose: For an adult, the recommended administered activity is $555-1,110 \mathrm{MBq}(15-30 \mathrm{mCi})$. Higher activities (up to $35 \mathrm{mCi}$ ) may be required for stress studies that are imaged using general/all-purpose 
collimators, or for obese patients. For children, the administered activity is $7-15 \mathrm{MBq} / \mathrm{kg}(0.2-$ $0.4 \mathrm{mCi} / \mathrm{kg}$ ), with a minimum dose of $70-150 \mathrm{MBq}$ (2-4 mCi). For pediatric patients, the EANM pediatric dosage card can be referred to for determination of weight based radioisotope dose (https://www.eanm.org/publications/dosagecalculator/). With the advent of cadmium-zinctelluride cameras, it is conceivable that doses will be significantly lower when compared with imaging with standard Anger cameras (16). Information regarding dosimetry is presented in Table 3 .

ii. Labeling: RBC labeling can be performed by 3 methods: In vivo technique with labeling efficiency of $60 \%-70 \%$; mixed in vivo/in vitro technique with labeling efficiency of $90 \%$; and in vitro with labeling efficiency above $97 \%$ (18-21). The mechanism of labeling is similar in all 3 methods.

Reduction of ${ }^{99 \mathrm{~m}} \mathrm{Tc}$-pertechnetate is required in order for it to bind firmly to hemoglobin. This is achieved by administration of stannous ions $\left(\mathrm{Sn}^{+2}\right)$ as approximately $10-20 \mu \mathrm{g} / \mathrm{kg}$ of stannous pyrophosphate, with free $\mathrm{Sn}^{+2}$ passively diffusing into the cell. ${ }^{99 \mathrm{~m}} \mathrm{Tc}$-pertechnetate is then added, 20-30 minutes after the injection of stannous pyrophosphate, which rapidly enters into the cells crossing the red cell membrane by diffusion or the band-3 anion transport system (22). Intracellular $\mathrm{Sn}^{+2}$ reduces intracellular pertechnetate that attaches to the $\beta$-chain of hemoglobin in the RBC. Reduction of pertechnetate outside the RBC (due to excess of free $\mathrm{Sn}^{+2}$ ) prevents it from entering the cell, resulting in reduced labeling efficiency. In vitro labeling can be performed with a commercially available kit (14). a. In vivo labeling: Intravenous injection of 10-20 $\mu \mathrm{g} / \mathrm{kg}$ of stannous pyrophosphate, followed by intravenous injection of ${ }^{99 \mathrm{~m}} \mathrm{Tc}$-pertechnetate after 20-30 minutes. Although this method results in a lower labeling efficiency, it results in satisfactory diagnostic quality. It eliminates the risk and requirements associated with handling of human blood. It is also more cost effective.

b. Mixed in vivo/vitro labeling: Injection of 10-20 $\mu \mathrm{g} / \mathrm{kg}$ of stannous pyrophosphate. After an in- terval of $20-30$ minutes, $3 \mathrm{~mL}$ of blood is drawn into a shielded syringe containing anticoagulant citrate dextrose and ${ }^{99 \mathrm{~m}}$ Tc-pertechnetate. Blood is allowed to incubate at least 10 minutes, and this radiolabeled blood is then reinjected for imaging.

c. In vitro labeling: Draw 1-3 mL of blood into a heparinized syringe/vial provided in the kit $(11,12)$. Add blood to the vial containing stannous chloride and incubate for 5-10 minutes. After this, sodium hypochlorite (syringe 1) is added to oxidize the extracellular stannous ion. Citric acid monohydrate and sodium citrate dehydrate (syringe 2) are subsequently added to sequester any residual extracellular stannous ion, rendering it more readily available for oxidation by sodium hypochlorite. This is followed by addition of ${ }^{99 \mathrm{~m}} \mathrm{Tc}$-pertechnetate to the vial, with reinjection of radiolabeled blood after 20 minutes.

2. Drug Interaction: Some drugs and devices may interact with the RBC labeling and normal tracer distribution (23-26). A representative list of drugs and medical conditions that adversely affect RBC labeling is provided in Table 4 (refer to section "VIII. Sources of Error \& Quality Assurance"). The use of a commercially available kit minimizes these alterations in RBC labeling. In general, tracer should not be injected through intravenous lines containing heparin, or through which heparin or dextrose containing solutions are being administered. Additionally, recent blood transfusion increases the level of circulating free hemoglobin, which will promote the formation of ${ }^{99 \mathrm{~m}} \mathrm{Tc}$ hemoglobin complex. As well, recent multiple blood transfusions may increase the formation of RBC antibody and affect the binding of the ${ }^{99 \mathrm{~m}}$ Tc-pertechnetate to the RBC. Furthermore, stannous ion may bind to the tubing in an in vivo labeling method.

\section{E. Image Acquisition $(5,11)$}

1. Planar ERNA

i. Instrumentation:

a. $\gamma$-camera (dual-head preferred) with ECG gating.

b. Collimators: For a rest-only acquisition, a lowenergy high-resolution (LEHR) (most common)

TABLE 3

Dosimetry

\begin{tabular}{|c|c|c|c|}
\hline 99mTc-labeled RBC (17) & Adult & Pediatrics-10 y old & Pediatrics -5 y old \\
\hline Urinary bladder (organ receiving the largest radiation dose) (mSv per MBq) & 0.012 & 0.0204 & 0.027 \\
\hline Effective dose (mSv per $\mathrm{MBq})$ & 0.0047 & 0.0077 & 0.011 \\
\hline \multicolumn{4}{|l|}{ 99mTc-radiolabeled human serum albumin (HSA) (17) } \\
\hline Heart (organ receiving the largest radiation dose) (mSv per MB)q & 0.0085 & 0.01 & 0.021 \\
\hline Effective dose (mSv per MBq) & 0.0046 & 0.007 & 0.011 \\
\hline
\end{tabular}


TABLE 4

Causes of Reduced RBC Labeling Efficiency

\begin{tabular}{|c|c|}
\hline Cause & Drug or process \\
\hline \multirow[t]{4}{*}{ Oxidation of stannous ion } & Hydralazine \\
\hline & Heparin \\
\hline & Methyldopa \\
\hline & Decreased hematocrit \\
\hline \multirow[t]{2}{*}{ Inadequate reduction of ${ }^{99 \mathrm{~m} T c}$} & Excess stannous chloride \\
\hline & $\begin{array}{l}\text { Insufficient stannous } \\
\text { chloride }\end{array}$ \\
\hline \multirow[t]{5}{*}{ Formation of RBC antibodies } & Methyldopa \\
\hline & Penicillin \\
\hline & Quinidine \\
\hline & Immune disorders \\
\hline & Leukemia and lymphoma \\
\hline Complex formation with ${ }^{99 m} \mathrm{Tc}$ & Dextrose \\
\hline \multirow[t]{3}{*}{ Decreased RBC labeling } & Prazosin \\
\hline & Digoxin \\
\hline & Propranolol \\
\hline \multirow[t]{2}{*}{ Mechanism unknown } & Doxorubicin \\
\hline & lodinated contrast \\
\hline (Adapted from (4)). & \\
\hline
\end{tabular}

or low-energy all-purpose (LEAP) can be used (4). A slant hole collimator can be used for better separation of the atria from the ventricles. For stress acquisition, LEAP is most commonly used. It is recommended that the same collimator be used for rest and stress studies.

c. Bicycle ergometer for an exercise protocol.

ii. Camera Setting:

a. Energy window: $140 \mathrm{keV}, \pm 10 \%$.

b. R-R beat acceptance window: For systolic function, a length of $\pm 10 \%-15 \%$ is commonly used. However, for patients with arrhythmias, the length may be increased.

c. Recording: Gated images should be stored in list mode if possible. With this method, the rejected and accepted beats are stored and can be used in patients with arrhythmias as well as to evaluate diastolic function. Alternatively, ERNA can be performed with arrhythmia beat rejection as well.

iii. Acquisition:

a. Zoom is adjusted so the heart occupies approximately $50 \%$ of the field of view.

b. A minimum of 16 frames per R-R interval is required (preferred rate of 24-32 frames per $R-R$ interval) for an appropriate evaluation of the ventricular function. For stress images, 32 frames per R-R beat is preferred for the evaluation of peak systolic and diastolic function.

c. Views: Three views are recommended for the most accurate assessment of the left ventricle wall motion (Fig. 1). EANM/ESC guidelines can be referred to regarding the specifics on chamber orientation and LV walls (11).
(1) The left anterior oblique (LAO) view (the best septal view) at $45^{\circ}$ or at an angle where the septum is best visualized is the recommended view for the separation of the left ventricle from the right. This is the most commonly used single view for evaluation of LVEF. Though a caudal tilt of $10^{\circ}-15^{\circ}$ may help in separating the atria from the ventricles, a cranial tilt can also be used to obtain appropriate chamber orientation. On a correctly angulated LAO view, the photopenic region of the interventricular septum is essentially verticalthat is, 12 o'clock to 6 o'clock orientation.

(2) Anterior view $\left(-45^{\circ}\right.$ more anterior from the LAO view) is used for the evaluation of the anterolateral wall and apex. In this projection, the apical, basal to mid anterolateral, and basal to mid inferoseptal walls of the left ventricle are seen. The RV blood-pool activity usually obscures the inferior wall.

(3) Lateral view $\left(+45^{\circ}\right.$ more lateral than the LAO) or left posterior oblique views can be used for the evaluation of the inferior basal to mid anterior walls. However, in this position, the LV images are interfered by the RV counts.

iv. Body Position: The distance between the camera and the patient should be as small as possible, based on patient body habitus.

a. Rest: Images are obtained in the supine position. Decubitus position may be used to decrease interference from the spleen and diaphragm. If stress images are to be acquired, the rest images are completed before stress imaging. Once the optimal position for the best septal view is determined, the rest anterior and lateral views are acquired.

b. Stress: Images can be acquired in a supine, upright, or sitting position while using the bicycle ergometer in one of the recommended views in multiple stages of exercise (LAO is preferred). At the completion of rest imaging, the patient is prepped for stress, including feet strapped in pedals; 12-lead ECG and blood pressure cuff is placed, etc., so the patient is not moved between the best

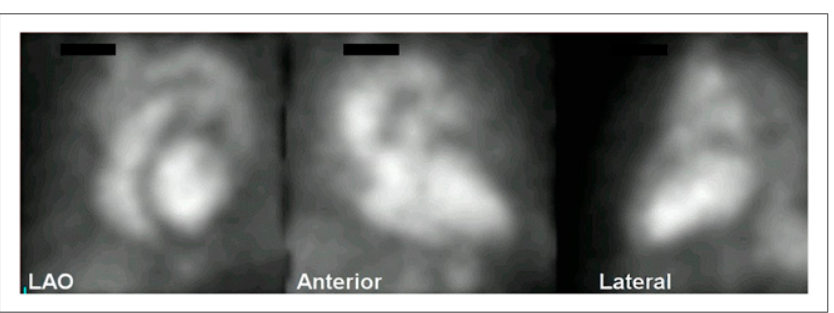

FIGURE 1. Standard views for ERNA. 


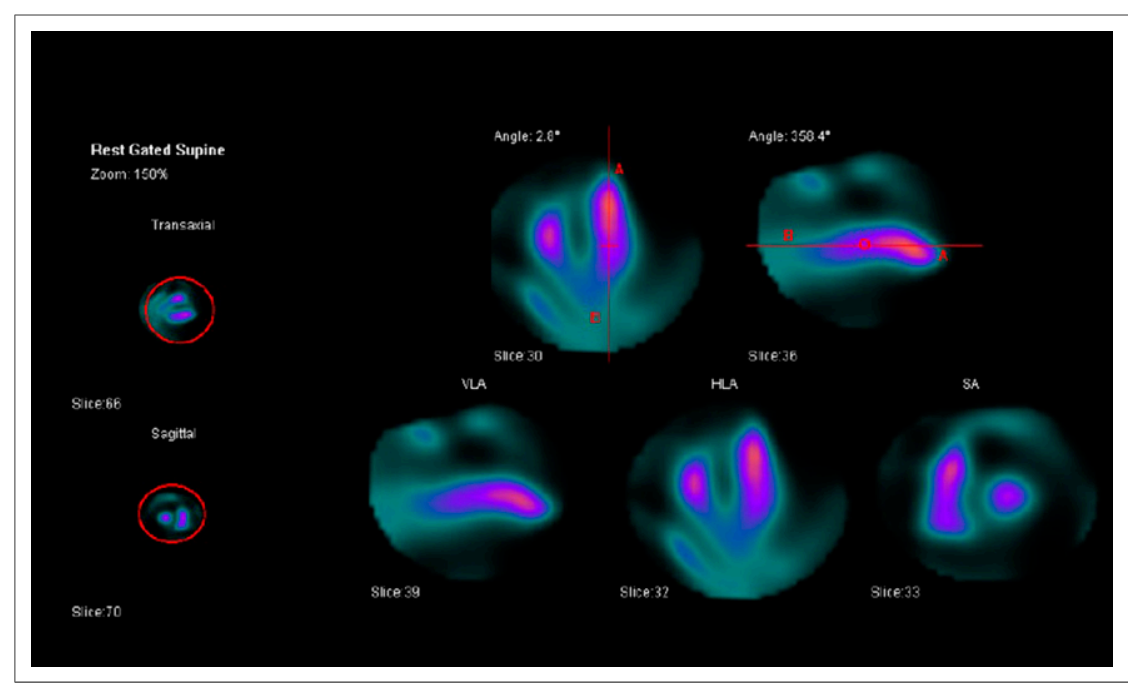

FIGURE 2. Example showing placement of ROls and orientation of the ventricles before processing of SPECT ERNA data. (Image courtesy of Ronald G. Schwartz, MD, University of Rochester Medical Center, Rochester, NY. Images acquired on a cadmium-zinc-telluride (CZT) camera.)

septal view acquired at rest and stress. Image acquisition typically begins approximately 1 minute into each exercise stage.

v. Counts: Overall the total of 3-7 million counts are typically acquired. 20,000 counts $/ \mathrm{cm}^{2}$ are typically generated with a high-resolution collimator and about 40,000 counts $/ \mathrm{cm}^{2}$ with a high-sensitivity collimator. Since count density may vary with size of the field of view, it is recommended that at least 200,000 counts per frame should be acquired for a 16-frame resting study using a high-resolution collimator. Since time is a limiting factor with stress images, at least 23 minutes of acquisition time at peak stress is recommended to obtain adequate count density.

\section{SPECT ERNA}

i. Instrumentation: Dual-head cameras are the most commonly used for $180^{\circ}$ acquisition orbit $\left(90^{\circ}\right.$ camera head orientation). Although much less common, triple-head or single-head cameras can be used for $180^{\circ}$ and $360^{\circ}$ image acquisition configurations. Cadmium-zinc-telluride cameras may decrease imaging time or radiopharmaceutical dose, while preserving study quality (16).

ii. Collimators: High-resolution parallel-hole collimators are preferred.

iii. Number of Projections:

a. Typically, for a $180^{\circ}$ image acquisition using dual-head SPECT cameras, 60-64 views (3032 per head) for 30 seconds per view is commonly used.

b. Triple-head-camera acquisition is similar to the dual-head-camera protocol.

c. With a single-head camera for $180^{\circ}$ image acquisition, 32 frames with 40-60 seconds per view are recommended. iv. Acquisition: 16 frames per $\mathrm{R}-\mathrm{R}$ interval is recommended. When both planar and SPECT ERNA are being performed, it is preferable to use the same $\mathrm{R}-\mathrm{R}$ beat parameters whenever possible.

v. SPECT reconstruction $(5,11)$ : Iterative reconstruction (if attenuation correction is performed) or filtered backprojection.

\section{IMAGE PROCESSING}

Assessment of LV functional parameters is performed by delineating regions of interest (ROIs). LVEF assessment by ERNA is count-based, and LV counts are measured from ROIs drawn over the LV, on the best septal separation view in end-diastole and end-systole. The LV counts are corrected for background counts (background correction [Bk-Corr]) obtained from an ROI placed adjacent to the lateral or the inferoapical wall of the LV (between the 2 o'clock and 5 o'clock position) (refer to section "VIII. Sources of Error \& Quality Assurance"). It is crucial to ensure that LV ROI does not include counts from other adjacent structures (such as RV, spleen, or aorta). LVEF is then calculated as:

$$
\begin{aligned}
& \text { Bk-Corr End }- \text { diastolic counts }- \\
& \frac{\text { Bk-Corr End }- \text { systolic counts }}{\text { Bk-Corr End }- \text { diastolic counts }} \times 100 \quad \text { Eq. } 1
\end{aligned}
$$

Contrary to ERNA, the 3-dimensional nature of SPECT image reconstruction obviates the need for background correction, thus allowing for volumetric assessment of LVEF by SPECT ERNA. Though count-based assessment can also be performed with SPECT ERNA, it is generally not recommended. ROIs are drawn to encompass the entire heart, with $\mathrm{LV}$ apex positioned at the 12 o'clock and 3 o'clock position on horizontal and vertical long axis views (Fig. 2). Most software automatically calculate LVEF without the need to individually draw end-diastolic and end-systolic ROIs. An output of the ventricular function data from SPECT ERNA is shown in Figure 3.

Images should be reviewed for overlapping of chambers that can alter ventricular counts and result in errors in calculation of LVEF. Additionally, time-activity curves generated from the ROIs should be assessed for frame drop-out that may result from variability in length of the cardiac cycle. This drop-out, which typically occurs in the diastolic frames, will lower the end-diastolic counts or volumes and result in underestimation of LVEF. Significant heart rate variability may also compromise the accurate determination of diastolic indices. 


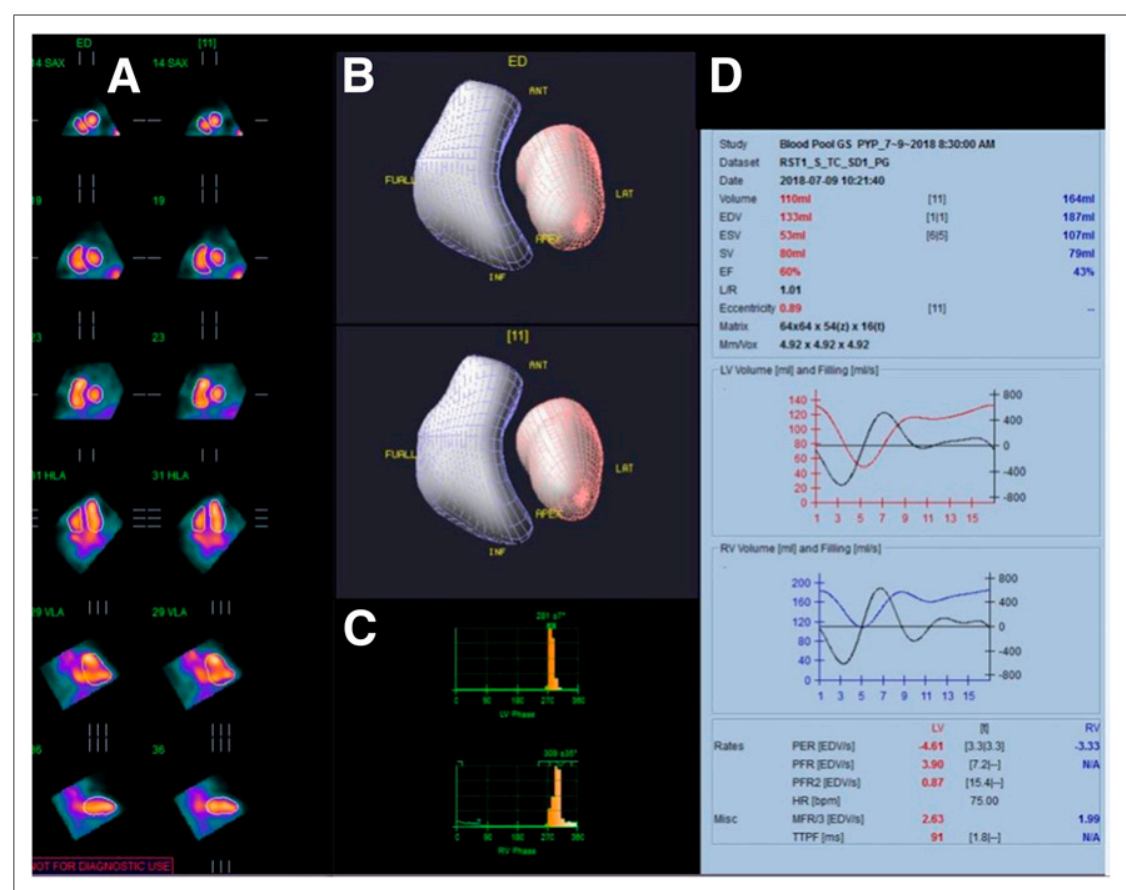

FIGURE 3. Ventricular function data from SPECT ERNA. SPECT ERNA data depicting end-diastolic and end-systolic contours (A), 3-dimensional rendering of LV and RV walls and morphology $(B)$, phase data $(C)$, and volumetric data for both ventricles (left in red and right in blue) (D). (Image courtesy of Ronald G. Schwartz, MD, University of Rochester Medical Center, Rochester, NY. Images acquired on a cadmium-zinc-telluride (CZT) camera.)

\section{IMAGE INTERPRETATION}

\section{A. Cardiac Morphology and Function}

The morphology, orientation, and sizes of the cardiac chambers and great vessels should be qualitatively evaluated. Qualitative assessment of LV and RV size should be performed, relative to each other, provided there is consistency in image acquisition across patients (camera type and magnification). Although quantitation of LVEF can be performed from planar ERNA, right ventricular ejection fraction (RVEF) cannot be reliably quantified (11). A more reliable assessment of biventricular volumes and RVEF can be performed with SPECT ERNA. Qualitative assessment of LV thickness and pericardial silhouette should be performed, to differen-

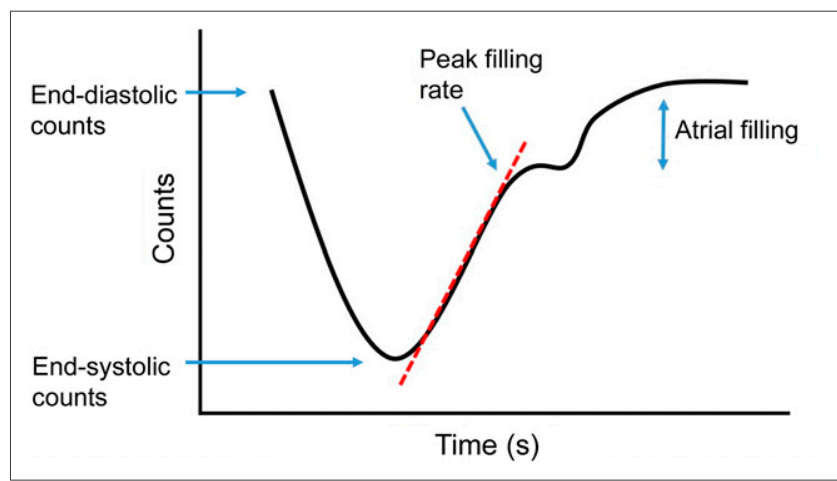

FIGURE 4. Example of a time-activity curve on ERNA. tiate LV hypertrophy from significant pericardial effusion. Qualitative assessment of the atrial sizes can be performed on the anterior (right atrium) and left lateral (left atrium) views.

A value of $\geq 50 \%$ is considered normal of LVEF (27). RVEF can be quantified by SPECT ERNA and while not systematically validated, a value of $\geq 40 \%$ is generally regarded as being the lower limit of normal (27). All LV segments should be assessed for regional function using cinematic display of each view. Abnormalities of contraction should be described using the conventional terms of normal, mild, moderate, or severe hypokinesis, akinesis and dyskinesis (28). Quantitative determination of ventricular volumes can be performed with SPECT ERNA, which is an advantage over planar ERNA. Good correlation of LVEF has been reported between SPECT ERNA and planar ERNA (29). However, when serial assessment is desired, it is recommended that the same technique for assessment of LVEF be used, for example, serial LVEF assessment for patients undergoing chemotherapy.

\section{B. Stress Images}

The stress or intervention study should be displayed sideby-side with the resting study in cinematic mode. Changes in chamber sizes, regional wall motion, and global ejection fraction of both ventricles should be addressed qualitatively

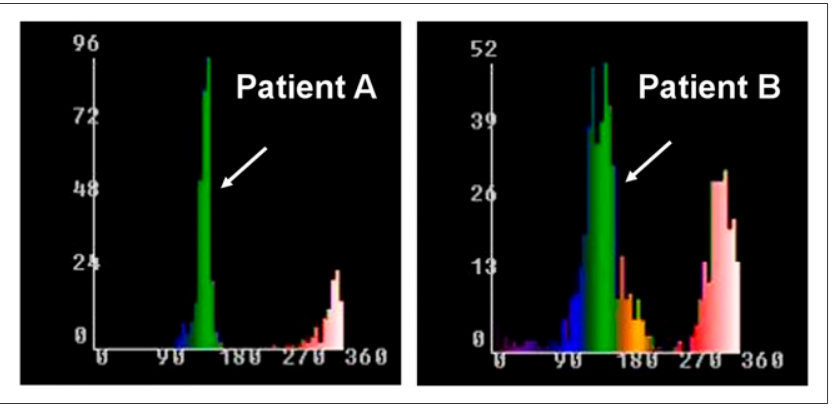

FIGURE 5. Assessment of LV dyssynchrony by phase analysis of ERNA. LV phase histograms (arrows) on phase analysis of ERNA from 2 patients depicting the timing of myocardial contraction ( $x$-axis in degrees) and the frequency of the pixels achieving contraction at a particular phase ( $y$-axis). Patient $A$ has a normal ejection of $70 \%$, with a uniform phase distribution and narrow phase histogram, depicting lack of dyssynchrony. Patient $B$ has a severely reduced ejection fraction of $30 \%$, with wide phase histogram, highlighting dyssynchronous LV contraction. 
TABLE 5

List of Clinically Relevant Data to be Included in an ERNA Report (33)

\begin{tabular}{|c|c|c|}
\hline Data & Planar ERNA & SPECT ERNA \\
\hline Demographic data & Standard & Standard \\
\hline Study date & Standard & Standard \\
\hline Medical record number & Standard & Standard \\
\hline Height/weight/BSA & Standard & Standard \\
\hline \multicolumn{3}{|l|}{ Acquisition information } \\
\hline Type of study & Standard & Standard \\
\hline Radionuclide and dose & Standard & Standard \\
\hline RBC Labeling technique & Standard & Standard \\
\hline Study indication & Standard & Standard \\
\hline Study quality & Recommended & Recommended \\
\hline \multicolumn{3}{|l|}{ Study results: rest } \\
\hline \multicolumn{3}{|l|}{ Left ventricle (LV) } \\
\hline \multicolumn{3}{|l|}{ LV size } \\
\hline Qualitative & Standard & Standard \\
\hline Quantitative (volume) & N/A & Optional \\
\hline LV regional wall motion & Standard & Standard \\
\hline LV hypertrophy & Optional & Optional \\
\hline Quantitative LVEF & Standard & Standard \\
\hline \multicolumn{3}{|l|}{ LV diastolic function } \\
\hline Qualitative & Standard & Standard \\
\hline Quantitative (PFR) & Preferred & Preferred \\
\hline \multicolumn{3}{|l|}{ Right ventricle (RV) } \\
\hline \multicolumn{3}{|l|}{ RV size } \\
\hline Qualitative & Standard & Standard \\
\hline Quantitative (volume) & N/A & Optional \\
\hline RV regional wall motion & Standard & Standard \\
\hline \multicolumn{3}{|l|}{ RVEF } \\
\hline Qualitative & Optional & Optional \\
\hline Quantitative* $^{\star}$ & N/A & Optional \\
\hline Atrial sizes & Optional & Optional \\
\hline Aorta and pulmonary artery size & Optional & Optional \\
\hline Pericardium & Optional & N/A \\
\hline Synchrony parameters & Optional & Optional \\
\hline \multicolumn{3}{|l|}{ Study results: stress } \\
\hline Type of protocol: exercise/intervention & Standard & Standard \\
\hline Symptoms & Standard & Standard \\
\hline Peak BP and HR & Standard & Standard \\
\hline METs or \%MPHR & Standard & Standard \\
\hline \multicolumn{3}{|l|}{ Left ventricle (LV) } \\
\hline \multicolumn{3}{|l|}{ LV size: change from rest } \\
\hline Qualitative & Standard & - \\
\hline Quantitative & $\mathrm{N} / \mathrm{A}$ & - \\
\hline Change in LV regional wall motion & Standard & - \\
\hline Quantitative stress LVEF & Standard & - \\
\hline \multicolumn{3}{|l|}{ Right ventricle $(\mathrm{RV})^{* *}$} \\
\hline RV size: change from rest & Standard & - \\
\hline Change in $\mathrm{RV}$ regional wall motion & Standard & - \\
\hline Qualitative stress RVEF & Optional & - \\
\hline \multicolumn{3}{|l|}{ Conclusion } \\
\hline Normal or abnormal & Standard & Standard \\
\hline Diagnostic significance of rest/stress response & Standard & - \\
\hline Prognostic significance of rest/stress response ${ }^{\star *}$ & Optional & - \\
\hline Comparison to prior studies & Standard & Standard \\
\hline Extracardiac findings & Recommended & Recommended \\
\hline
\end{tabular}

${ }^{\star}$ RVEF can be obtained from SPECT ERNA, though not well validated.

${ }^{* *}$ Pharmacologic stress SPECT ERNA can allow for assessment of these parameters, though there is little contemporary data.

$\mathrm{BSA}$ = body surface area; LVEF = left ventricular ejection fraction; RVEF = right ventricular ejection fraction; PFR = peak filling rate; $\mathrm{BP}=$ blood pressure; $\mathrm{HR}=$ heart rate. 


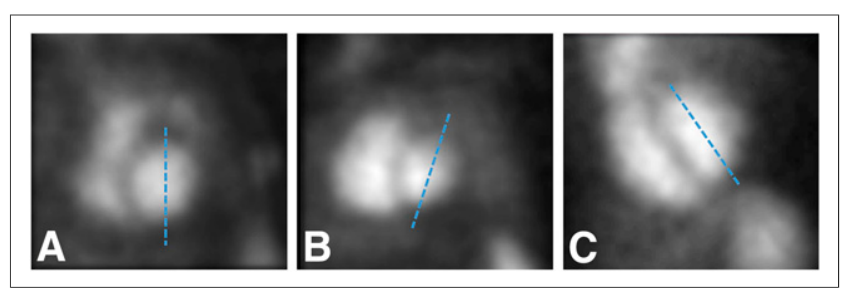

FIGURE 6. Correct orientation of the left ventricle in the LAO view. (A) Optimal LAO orientation, with a vertical LV axis (dashed line). Suboptimal LV axis with rightward tilting (B) and leftward tilting $(\mathrm{C})$ of the LV axis.

and reported with quantitative measures of ejection fraction. Normal stress LVEF should increase by at least $5 \%$ or $5 \mathrm{EF}$ units from rest.

\section{LV Diastolic Function}

The pattern of LV diastolic function can be evaluated qualitatively from the time-activity curve (Fig. 4). It can also be quantified as the peak filling rate (PFR). In cardiomyopathy, even before impairment of systolic function, there is slower and later than normal filling of the LV. This is secondary to poor LV compliance and reflects diastolic dysfunction. Presence of diastolic dysfunction in the absence of overt systolic dysfunction could also be indicative of occult coronary artery disease. Thus, PFR, which can often be reduced before a reduction in LVEF, allows for identification of a cardiomyopathic process before measurable impairment of LV function. A PFR of $>2.5$ end-diastolic volume (counts) per second (EDV/s) is considered normal on planar ERNA (4). While there is good correlation between planar and SPECT ERNA with respect to LVEF assessment, there is only a modest to weak correlation between measures of diastolic function (1).

\section{Assessment of LV Dyssynchrony}

LV dyssynchrony is a state of disorganized ventricular contraction, wherein there is a temporal dispersion in the activation of the ventricle. First harmonic phase analysis of the time-activity curve from each pixel in ERNA allows for the determination of the mean and SD of LV phase $(\phi)$. The $\phi$ distribution from various LV pixels is represented as a phase histogram (Fig. 5) that allows for qualitative assessment of LV dyssynchrony. Objective measures of LV dyssynchrony, the synchrony $(S)$ and entropy $(E)$ parameters, have been developed from planar ERNA. $S$ expresses the efficiency of contraction within an ROI, whereas $E$ measures the degree of randomness within the ROI, from 0 , with synchronous motion and a single $\phi$, to 1 with fully dyssynchronous contraction. Normal values of these parameters have been reported and are $59.4^{\circ}$ for SD of $\phi$, 0.73 for $S$, and 0.77 for $E$. These measures of LV dyssynchrony have been shown to improve patient selection for cardiac resynchronization therapy (CRT), predict and quantitate CRT outcomes, optimize CRT pacemaker lead placement based on location of the latest contracting segment, and have also been applied to measure RV synchrony (30-32).

\section{E. Comparison with Previous Studies}

Results should be compared with any previous studies by direct comparison of the cinematic displays of the 2 studies, whenever possible, and should be stated in the final report. Given the excellent reproducibility of serial ERNA examinations of $<5 \%$, a $5 \%$ change in LVEF would imply a significant change between 2 examinations.

\section{IMAGE REPORTING}

Use of a standard template is highly recommended to ensure that all clinically relevant data elements are accurately reported and to allow ease of interpretation for the referring provider. A detailed list of clinically relevant elements of a complete ERNA report is provided in Table 5.

\section{SOURCES OF ERROR \& QUALITY ASSURANCE}

\section{A. RBC Labeling}

Certain medications and disease processes will decrease labeling efficiency and reduce the target-to-background ratio. Common causes of reduced RBC labeling efficiency are provided in Table 4.

\section{B. Patient and Camera Positioning}

Suboptimal orientation of the best septal separation view (LAO orientation) can result in inaccurate calculation of LVEF. This is especially true when there is overlap of the LV by other cardiac structures (Fig. 6), for example, overlap with the left atrium, which has a time-activity curve that is the opposite of that of the left ventricle. Patient motion may also result in significant artifacts.

\section{Attenuation}

The left breast can attenuate the heart, especially in the LAO view. This may give an illusion of a halo around the heart, and care should be taken during image interpretation as this can be confused with a pericardial effusion.

\section{Gating Errors}

A poor ECG signal or one in which complexes other than the QRS complex are dominant may result in spurious gating, with functional data that are not interpretable. Care should be taken to ensure that the QRS complex is the triggering signal (e.g., choosing an ECG lead in which the QRS is much larger than the T wave). The best gating can

FIGURE 7. Location of LV and background $\mathrm{ROI}$ for calculation of LV function. Blue oval $=$ LV ROI; yellow oval $=$ correct location of background $\mathrm{ROI}$; red oval = incorrect location of background $\mathrm{ROI}$ (over the spleen).

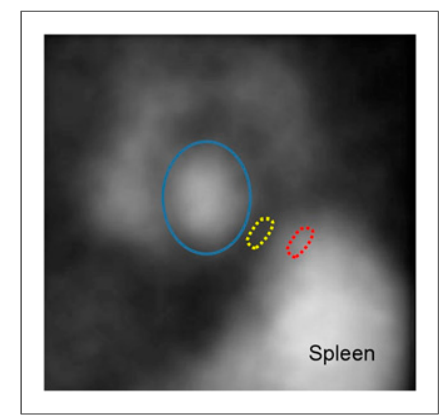


be obtained from systems that compute the rate of change of voltage on the ECG to be sure that the QRS and not the T wave is the signal used. Beat length R-R interval histogram should be assessed to determine heart rate variability and any rhythm abnormalities. Typical beat length acceptance window for planar ERNA is the mean R-R interval $\pm 10 \%-$ $15 \%$ and $\pm 15 \%-35 \%$ for SPECT ERNA, but will vary depending on heart rate and rhythm (2). Though gating errors affect both systolic and diastolic parameters, the errors are more severe for diastolic parameters.

\section{E. Image Statistics}

Inadequate counts/frames may compromise image interpretation as well as decrease the statistical reliability of quantitative measurements. Additionally, adjacent high liver and spleen activity may corrupt the count statistics of ventricular ROIs. Image display normalization to the peak activity in the chamber of interest can reduce the scatter effects of intense activity in the adjacent tissues.

\section{F. Processing Errors}

Inclusion of extraventricular activity or exclusion of ventricular activity from ventricular ROIs may cause underestimation or overestimation of the EF. Including the left atrium in the ROI will also lower the LVEF. Inclusion of structures such as the spleen or the descending aorta in the background ROI may alter the LVEF. This would result in an increase in the background counts and a resultant overestimation of LVEF (Fig. 7). Placing the background ROI outside the body will result in an underestimation of LVEF.

\section{BIBLIOGRAPHY}

1. Martin RJ, Santiago B. Left ventricular function parameters in a Hispanic population: comparison of planar \& tomographic radionuclide ventriculography (MUGA). P R Health Sci J. 2015;34:155-158.

2. Jensen MM, Schmidt U, Huang C, Zerahn B. Gated tomographic radionuclide angiography using cadmium-zinc-telluride detector gamma camera; comparison to traditional gamma cameras. J Nucl Cardiol. 2014;21:384-396.

3. Klocke FJ, Baird MG, Lorell BH, et al. ACC/AHA/ASNC guidelines for the clinical use of cardiac radionuclide imaging: executive summary - a report of the American College of Cardiology/American Heart Association Task Force on Practice Guidelines (ACC/AHA/ASNC Committee to Revise the 1995 Guidelines for the Clinical Use of Cardiac Radionuclide Imaging). J Am Coll Cardiol. 2003;42:1318-1333.

4. Corbett JR, Akinboboye OO, Bacharach SL, et al. Equilibrium radionuclide angiocardiography. J Nucl Cardiol. 2006;13:e56-e79.

5. Hesse B, Tagil K, Cuocolo A, et al. EANM/ESC procedural guidelines for myocardial perfusion imaging in nuclear cardiology. Eur J Nucl Med Mol Imaging. 2005;32:855-897.

6. Sachpekidis C, Sachpekidis V, Kopp-Schneider A, Arsos G, Moralidis E. Equilibrium radionuclide angiography: Intra- and inter-observer repeatability and reproducibility in the assessment of cardiac systolic and diastolic function. $\mathrm{J} \mathrm{Nucl}$ Cardiol. August 5, 2019 [Epub ahead of print].

7. van der Vleuten PA, Slart RH, Tio RA, et al. The feasibility of repeated left ventricular ejection fraction analysis with sequential single-dose radionuclide ventriculography. Nucl Med Commun. 2005;26:711-715.

8. van Royen N, Jaffe CC, Krumholz HM, et al. Comparison and reproducibility of visual echocardiographic and quantitative radionuclide left ventricular ejection fractions. Am J Cardiol. 1996;77:843-850.

9. Schwartz RG, McKenzie WB, Alexander J, et al. Congestive heart failure and left ventricular dysfunction complicating doxorubicin therapy: seven-year expe- rience using serial radionuclide angiocardiography. Am J Med. 1987;82:11091118.

10. Russell RR, Alexander J, Jain D, et al. The role and clinical effectiveness of multimodality imaging in the management of cardiac complications of cancer and cancer therapy. J Nucl Cardiol. 2016;23:856-884.

11. Hesse B, Lindhardt TB, Acampa W, et al. EANM/ESC guidelines for radionuclide imaging of cardiac function. Eur J Nucl Med Mol Imaging. 2008;35:851-885.

12. Cardiac function/ERNA. In: Hustinx R, Muylle K, eds. European Nuclear Medicine Guide. Vienna, Austria: European Association of Nuclear Medicine and Nuclear Medicine Section of the European Union of Medical Specialists; 2018: https://www.nucmed-guide.app/\#!/home. Accessed May 13, 2020: chapter 2.3.

13. Henzlova MJ, Duvall WL, Einstein AJ, Travin MI, Verberne HJ. ASNC imaging guidelines for SPECT nuclear cardiology procedures: Stress, protocols, and tracers. J Nucl Cardiol. 2016;23:606-639.

14. Verberne HJ, Acampa W, Anagnostopoulos C, et al. EANM procedural guidelines for radionuclide myocardial perfusion imaging with SPECT and SPECT/ CT: 2015 revision. Eur J Nucl Med Mol Imaging. 2015;42:1929-1940.

15. Nagaoka H, Isobe N, Kubota S, et al. Comparison of adenosine, dobutamine, and exercise radionuclide ventriculography in the detection of coronary artery disease. Cardiology. 1997;88:180-188.

16. Chen YC, Ko CL, Yen RF, et al. Comparison of biventricular ejection fractions using cadmium-zinc-telluride SPECT and planar equilibrium radionuclide angiography. J Nucl Cardiol. 2016;23:348-361.

17. Stabin MG, Siegel JA. RADAR dose estimate report: a compendium of radiopharmaceutical dose estimates based on OLINDA/EXM version 2.0. J Nucl Med. 2018;59:154-160

18. Pavel DG, Zimmer M, Patterson VN. In vivo labeling of red blood cells with ${ }^{99 \mathrm{~m} T \mathrm{~T}:}$ a new approach to blood pool visualization. J Nucl Med. 1977;18:305-308.

19. Callahan RJ. Radiolabeled red blood cells: method and mechanisms. The University of New Mexico Health Sciences Center College of Pharmacy Accreditation Council for Pharmacy Education ACPE; 2006. https://pharmacyce.unm.edu/ nuclear_program/freelessonfiles/Vol12Lesson1.pdf. Accessed May 15, 2020.

20. Bauer R, Haluszczynski I, Langhammer H, Bachmann W. In vivo/in vitro labeling of red blood cells with ${ }^{99 m}$ Tc. Eur J Nucl Med. 1983;8:218-222.

21. UltraTag RBC [FDA package insert]. Rockville, MD: Mallinckrodt Inc.; 2012.

22. Callahan RJ, Rabito CA. Radiolabeling of erythrocytes with technetium-99m: role of band-3 protein in the transport of pertechnetate across the cell membrane. J Nucl Med. 1990;31:2004-2010.

23. Kawabe J, Higashiyama S, Torii K, et al. Poor labeling of Tc-99m red blood cells in vivo in a radionuclide intestinal bleeding study of a patient who had recently undergone frequent blood transfusions. Clin Nucl Med. 2003;28:911912.

24. Hladik WB, 3rd, Nigg KK, Rhodes BA. Drug-induced changes in the biologic distribution of radiopharmaceuticals. Semin Nucl Med. 1982;12:184-218.

25. Zou X, Duan XC, Zhong Q. Alteration of anterior chamber in 81 glaucomatous eyes using Pentacam Scheimpflug system. Int $J$ Ophthalmol. 2010;3:349-351.

26. Sampson CB. Complications and difficulties in radiolabelling blood cells: a review. Nucl Med Commun. 1996;17:648-658.

27. Pfisterer ME, Battler A, Zaret BL. Range of normal values for left and right ventricular ejection fraction at rest and during exercise assessed by radionuclide angiocardiography. Eur Heart J. 1985;6:647-655.

28. Cerqueira MD, Weissman NJ, Dilsizian V, et al. Standardized myocardial segmentation and nomenclature for tomographic imaging of the heart. A statement for healthcare professionals from the Cardiac Imaging Committee of the Council on Clinical Cardiology of the American Heart Association. J Nucl Cardiol. 2002; 9:240-245.

29. Massardo T, Jaimovich R, Lavados H, et al. Comparison of radionuclide ventriculography using SPECT and planar techniques in different cardiac conditions. Eur J Nucl Med Mol Imaging. 2007;34:1735-1746.

30. Singh H, Singhal A, Sharma P, Patel CD, Seth S, Malhotra A. Quantitative assessment of cardiac mechanical synchrony using equilibrium radionuclide angiography. J Nucl Cardiol. 2013;20:415-425.

31. Johnson CJ, Roberts JD, James JH, et al. Comparison of radionuclide angiographic synchrony analysis to echocardiography and magnetic resonance imaging for the diagnosis of arrhythmogenic right ventricular cardiomyopathy. Heart Rhythm. 2015;12:1268-1275.

32. Soman P. Radionuclide imaging in heart failure. In: Zaret BL BG, ed. Clinical Nuclear Cardiology, State of the Art and Future Directions. 4th ed. Oxford, U.K.: Mosby Elsevier; 2010.

33. Tilkemeier PL, Bourque J, Doukky R, Sanghani R, Weinberg RL. ASNC imaging guidelines for nuclear cardiology procedures: Standardized reporting of nuclear cardiology procedures. J Nucl Cardiol. 2017;24:2064-2128. 